\title{
Hemodynamic variations between continuous paravertebral and epidural thoracic block in laparoscopic esophagectomy : a prospective study.
}

P. Colombel1, A. Lazkani1, C. Gontier1, G. Lebuffe1

\section{Background and Goal of Study:}

Recently the paravertebral thoracic catheter (PVCT) was developed with ultrasounds techniques in thoracic surgery and it seems that it induces less hypotension than epidural (ET) with similar quality of analgesia. The purpose of this study is to compare, for the first time, the proportion of the hypotension episode in both groups in the esophagus surgery, Lewis-Santy, with thoracotomy and laparoscopy

\section{Materials and methods:}

Prospective, randomized, interventional, monocentric study.

After ethical committee approval, 26 patients in ET group vs. 28 in PVCT group were included.

$>$ They had both the following protocol: A bolus of $5 \mathrm{ml}$ of Ropivacaïne $0,2 \%$ and $10 \mu \mathrm{g}$ of Sufentanil was injected at the end of the abdominal part of the surgery, then an infusion of Ropivacaïne $0,2 \% 4 \mathrm{ml} / \mathrm{h}$ was realized at the thoracic time. Postoperative analgesia consisted on a Ropivacaïne $0,2 \%$ infusion at $6 \mathrm{ml} / \mathrm{h}$ in the ET groupe and $8 \mathrm{ml} / \mathrm{h}$ in the PVCT group and a bolus of $4 \mathrm{ml}$ every $15 \mathrm{~min}$ as required.

The primary endpoint was a mean arterial pressure (MAP) less than $70 \mathrm{mmHg}$ or a decrease in MAP of about $20 \%$ when compared to preopérative one. Hypotension was correlated to PVCT or ET when all other surgical or anesthesic causes of hypotensions was verified by a standard algorithm in both groups. We compared the MAP till 15 min after the bolus, during the thoracic time, and in the postoperative period until 48 hours.

Secondary endpoints was: fluid administration, the use of vasopressor drugs, the analgesic efficacy (NRS), and the use of morphine during the post-operative time till 48 hours in both groups. Chronic pain at 3 and 6 months was also compared.

\section{Results:}

There were significant differences between the two groups regarding the episodes of hypotension $(23 / 26)$ in ET group vs. $(11 / 28)$ in PVCT group $(p<0,001)$.

$>$ The proportion of hypotension was significantly higher after the bolus $(p=0,002)$ and on post operative period $(p<0,001)$.

$>$ We noticed more use of ephedrine in perioperative $(p=0,019)$ and of noradrenaline in postoperative ( $\mathrm{p} 4$, morphine consumption, and chronic pain.

\section{Conclusion:}

PVCT provides better hemodynamic stability than ET, but it is equal efficacy on analgesia to ET in esophagus surgery 